\title{
Aspirin unresponsiveness predicts thrombosis in high-risk pediatric patients after cardiac surgery
}

\author{
Sirisha Emani, PhD, Bethany Trainor, RN, David Zurakowski, PhD, Christopher W. Baird, MD, \\ Francis E. Fynn-Thompson, MD, Frank A. Pigula, MD, and Sitaram M. Emani, MD
}

\begin{abstract}
Objective: Thrombosis occurs in up to $26 \%$ of patients with congenital heart disease after cardiac surgery and is associated with increased morbidity and mortality. Aspirin is commonly administered to reduce the risk of thrombosis, yet aspirin responsiveness is rarely assessed. In this study, we hypothesize that inadequate response to aspirin is associated with increased risk of thrombosis after selected congenital cardiac procedures considered to be high risk for thrombosis.
\end{abstract}

\begin{abstract}
Methods: Patients undergoing high-risk congenital cardiac surgery who received postoperative aspirin $(\mathrm{N}=95)$ were studied. Response to aspirin was determined using the VerifyNow system several days after administration. Patients were monitored prospectively for 30 days for the development of a thrombosis event and the relationship between aspirin unresponsiveness and a thrombosis event was determined by the Fisher exact test.
\end{abstract}

Results: Rate of aspirin unresponsiveness ( $\geq 550$ aspirin reaction units [ARU]) was 10 of $95(10.5 \%)$ and was highest in patients weighing less than $5 \mathrm{~kg}$ given $20.25 \mathrm{mg} / \mathrm{d}$ of aspirin. Thrombosis events occurred in 7 patients (7.4\%). Thrombosis was observed in 6 of $10(60 \%)$ patients who were unresponsive to aspirin, compared with 1 of $85(1.2 \%)$ patients who were responsive to aspirin $(P<.001)$. In 2 patients who were unresponsive to the initial aspirin dose, an increase in dose resulted in an adequate therapeutic aspirin response (ARU $<550)$, suggesting insufficiency rather than true resistance in a subset of patients.

Conclusions: Postoperative thrombosis is associated with aspirin unresponsiveness in this patient population. In high-risk patients, monitoring of aspirin therapy and consideration of dose adjustment or alternative agents for unresponsive patients may be justified and warrants further investigation in a prospective trial. (J Thorac Cardiovasc Surg 2014;148:810-6)

Pediatric patients undergoing cardiac surgery are at higher risk for thrombosis than the general pediatric population. ${ }^{1,2}$ Thrombosis rates reported in the literature range from $10 \%$ to $20 \%$; the risk factors for thrombosis include young age, single ventricle circulation, and duration of central venous catheters. ${ }^{3-6}$ Antiplatelet therapy with aspirin is commonly used to reduce thrombosis after high-risk cardiac surgical procedures that require either insertion of prosthetic material or prosthetic valves into the circulation or significant reconstruction of coronary arteries.

Aspirin resistance has been reported in up to $26 \%$ of pediatric patients with cardiovascular defects undergoing surgical procedures, and significant interpatient variability in response to a particular dose of aspirin exists. ${ }^{7}$ Unpredictable aspirin responsiveness in pediatric patients may be

From Boston Children's Hospital, Boston, Mass.

This work was supported by an Elliott Field Family Fund.

Disclosures: Authors have nothing to disclose with regard to commercial support.

Read at the 94th Annual Meeting of The American Association for Thoracic Surgery, Toronto, Ontario, Canada, April 26-30, 2014.

Received for publication April 9, 2014; revisions received June 4, 2014; accepted for publication June 10, 2014.

Address for reprints: Sitaram M. Emani, MD, 300 Longwood Ave, Building: 273

Bader, Boston, MA 02115 (E-mail: sitaram.emani@cardio.chboston.org).

0022-5223/\$36.00

Copyright (c) 2014 by The American Association for Thoracic Surgery

http://dx.doi.org/10.1016/j.jtcvs.2014.06.016 caused by either inadequate dosing or true pharmacologic resistance. Measurement of platelet inhibition confirms the efficacy of aspirin, but is rarely performed in this population. This study tests the hypothesis that significant interpatient variability in aspirin responsiveness exists with the current dosing regimen used at our institution. Furthermore, we hypothesize that, among patients considered to be at high risk for thrombosis, the rate of thrombosis events is higher in patients who are unresponsive to aspirin compared with those who are responsive.

\section{METHODS \\ Patients and Study Design}

Pediatric and young adult patients with congenital heart disease (age $<18$ years) undergoing cardiac surgery at Boston Children's Hospital between January 1, 2013, and May 10, 2014, who were considered to be at high risk for thrombosis and deemed appropriate for aspirin therapy were enrolled into a prospective observation study after initiation of aspirin. Varying doses of aspirin $(20.25,40.5$, or $81 \mathrm{mg} / \mathrm{d})$ were administered orally or via nasogastric tube after postoperative bleeding complications were stabilized. Patients considered to be at high risk for thrombosis included those undergoing implantation of prosthetic material into the circulation (Blalock-Taussig shunt, stage 1 palliation, Fontan procedure, intracardiac baffles) and those undergoing coronary artery reconstruction (arterial switch operation, coronary artery unroofing, or reimplantation procedures). Exclusion criteria included: (1) coadministration of additional antiplatelet agents (clopidogrel, prasugrel); (2) inability to collect a blood sample; and (3) documented thrombosis before initiation of aspirin therapy. This 


\section{Abbreviations and Acronyms \\ $\mathrm{ARU}=$ aspirin reaction units \\ $\mathrm{IQR}=$ interquartile range}

prospective study was approved by the Boston Children's Hospital Institutional Review Board and signed consent was obtained.

\section{VerifyNow Aspirin Platelet Function Testing}

Aspirin responsiveness was measured using the VerifyNow system (Accumetrics, San Diego, Calif) for quantitative measurement of platelet aggregation. This test incorporates activation of platelets in a sample of whole blood by addition of an agonist, arachidonic acid, and measures platelet aggregation to fibrinogen-coated beads in a premade testing cassette. Platelet aggregation leads to increased light transmittance recorded as aspirin reaction units (ARU). Values less than 550 ARU indicate aspirin responsiveness; values of $550 \mathrm{ARU}$ or higher indicate unresponsiveness.

Platelet testing was performed after at least 2 doses of aspirin were administered to the patients. A sample of whole blood $(2 \mathrm{~mL})$ was drawn into a $3.2 \%$ sodium citrate vacuette tube (Greiner Bio-One, Monroe, $\mathrm{NC}$ ) by direct venipuncture or from an indwelling catheter after collection of at least $5 \mathrm{~mL}$ of blood, and was incubated for at least 30 minutes (but no longer than 2 hours) at room temperature according to the manufacturer's recommendations. The sample was then loaded into the VerifyNow cartridge and analyzed by the system.

\section{Assessment of a Thrombosis Event}

Patients were followed prospectively to detect the occurrence of a thrombosis event within 30 days of initiation of aspirin. Weekly review of clinical records and imaging studies as well as direct interaction with care providers were used to detect thrombosis events. An echocardiogram was obtained within 2 weeks after surgery or before discharge in all patients according to standard clinical care protocol. Clinical thrombosis events (stroke, shunt thrombosis, limb ischemia) and evidence of thrombosis by imaging studies (echocardiography or cardiac catheterization) were recorded, and the interval to the event was documented. Variables believed to contribute to the risk of thrombosis (age, concomitant use of heparin) were also recorded.

\section{Statistical Analysis}

Distribution of continuous variables are represented as the median with the interquartile range (IQR) and comparisons were made using the independent Student $t$ test. Categorical variables are expressed as percentages and compared using the Fisher exact test. Multivariate analysis was used to identify independent predictors of aspirin unresponsiveness and thrombosis. All statistical tests were 2-tailed.

\section{RESULTS \\ Patient Characteristics}

Although 110 patients met the initial inclusion criteria, only 95 were included in the study because of the inability to collect blood samples or the occurrence of a thrombosis event before initiation of aspirin. The general characteristics of the study population are shown in Table 1. Cardiac surgical procedures performed on patients given aspirin therapy in the postoperative period are listed in Table 1.

Median time from day of surgery to aspirin administration was 4 days with an IQR of 3 to 7 days. Aspirin testing was performed at a median of 4 days (IQR, 2-7 days) after initiation of aspirin. The median dose of aspirin administered was $6.5 \mathrm{mg} / \mathrm{kg} / \mathrm{d}$ (IQR, 4.2-9 mg/kg/d). Although most of the patients received heparin in the immediate postoperative period, 28 of 95 patients $(29.5 \%)$ were on heparin at the time of platelet testing. Unresponsiveness to aspirin therapy, defined as more than 549 ARU, was present in 10 of $95(10.5 \%)$ patients. The rate of thrombosis after surgery in this cohort was 7 of 95 $(7.4 \%)$, and the location of the thrombosis for each patient is listed in Table 1.

\section{Effect of Aspirin Dose on Unresponsiveness}

Aspirin dose administered in this cohort of patients was $20.25,40.5$, or $81 \mathrm{mg} / \mathrm{d}$. The median age, weight and number of patients categorized by dosage are shown in Table 2 . Of the 7 patients given $20.25 \mathrm{mg} / \mathrm{d}, 4(57.1 \%)$ were unresponsive to aspirin therapy. All patients in this group were neonates less than 1 month old and weighed less than $5 \mathrm{~kg}$. For patients receiving 40.5 and $81 \mathrm{mg} / \mathrm{d}$ of aspirin, 4 of $50(8 \%)$ and 2 of $38(5.3 \%)$ were unresponsive to aspirin therapy, respectively. Logistic regression indicated a significantly higher unresponsiveness rate for 20.25 $\mathrm{mg} / \mathrm{d}$ versus $40.5 \mathrm{mg} / \mathrm{d}(57.1 \%$ vs $8 \% ; P=.003)$ and $20.25 \mathrm{mg} / \mathrm{d}$ versus $81 \mathrm{mg} / \mathrm{d}(57.1 \%$ vs $5.3 \% ; P=.002)$ but no difference between $40.5 \mathrm{mg} / \mathrm{d}$ versus $81 \mathrm{mg} / \mathrm{d}(8 \%$ vs $5.3 \% ; P=.56$ ). In 2 patients who were unresponsive to an initial aspirin dose of 20.25 and $40.5 \mathrm{mg} / \mathrm{d}$, increasing the dose to 40.5 and $81 \mathrm{mg} / \mathrm{d}$, respectively, resulted in an adequate response, suggesting insufficiency rather than true resistance in this subset of patients.

\section{Relationship Between Aspirin Unresponsiveness and Thrombosis}

Thrombosis was observed in 7 of $95(7.4 \%)$ patients in this study. Thrombosis developed in 2 of $7(28.6 \%)$ patients who received $20.5 \mathrm{mg} / \mathrm{d}, 3$ of $50(6 \%)$ patients who received $40.5 \mathrm{mg} / \mathrm{d}$, and 2 of $38(5.3 \%)$ patients who were given $81 \mathrm{mg} / \mathrm{d}$ (Table 2). Logistic regression indicated a significantly higher rate of thrombosis for $20.25 \mathrm{mg} / \mathrm{d}$ versus $81 \mathrm{mg} / \mathrm{d}(28.6 \%$ vs $5.3 \% ; P=.025)$; no significant differences was observed between $40.5 \mathrm{mg} / \mathrm{d}$ versus $81 \mathrm{mg} / \mathrm{d}(6.1 \%$ vs $5.3 \% ; P=.69)$. There was a trend toward a difference in the rates of thrombosis between patients given $20.25 \mathrm{mg} / \mathrm{d}$ versus $40.5 \mathrm{mg} / \mathrm{d}(28.6 \%$ vs $6 \%$; $P=.06$ ); this difference was not statistically significant.

Thrombosis occurred more commonly in nonresponders compared with responders (Figure 1). Only 1 of 85 responders had a thrombosis event after the administration of aspirin, whereas 6 of 10 nonresponders had a thrombosis $(1.2 \%$ vs $60 \%$, respectively; $P<.001)$. Multivariate logistic regression analysis indicated that, after adjusting for age $(P=.04)$ and weight $(P=.76)$, aspirin dose was an independent predictor of unresponsiveness $(P<.01)$ 
TABLE 1. Patient characteristics

\begin{tabular}{|c|c|}
\hline Variable & Data \\
\hline Total number of patients & 95 \\
\hline Male, n (\%) & $55(57.9)$ \\
\hline Age & $1.1 \mathrm{y}(2.8 \mathrm{mo}$ to $3.1 \mathrm{y})$ \\
\hline Weight $(\mathrm{kg})$ & $8.15(4.2-13.9)$ \\
\hline Platelet count $\times 1000$ cells (per $\mu \mathrm{L}$ ) & $310(215-455)$ \\
\hline Heparin at time of testing, $\mathrm{n}(\%)$ & $28(29.5)$ \\
\hline Aspirin dose (mg/d) & $40.5(20.25-81)$ \\
\hline Aspirin dose (mg/kg/d) & $6.5(4.2-9)$ \\
\hline Date of surgery to aspirin administration, $d$ & $4(3-7)$ \\
\hline Duration on aspirin before testing, $\mathrm{d}$ & $4(2-7)$ \\
\hline Thrombosis events, n (\%) & $7(7.4)$ \\
\hline \multicolumn{2}{|l|}{ Surgical procedures, $\mathrm{n}$} \\
\hline Complex valve procedures & 27 \\
\hline Fontan & 15 \\
\hline Baffle & 8 \\
\hline Stage 1 palliation & 8 \\
\hline Arterial switch operation & 7 \\
\hline Blalock-Taussig shunt & 6 \\
\hline Tetralogy of Fallot & 5 \\
\hline Bidirectional Glen & 6 \\
\hline Coronary artery unroofing & 4 \\
\hline TAPVR & 3 \\
\hline Extensive branch PA reconstruction & 1 \\
\hline Complex LVOT reconstruction & 4 \\
\hline ALCAPA coronary artery repair & 1 \\
\hline \multicolumn{2}{|l|}{ Thrombosis location, $\mathrm{n}$} \\
\hline $\begin{array}{l}\text { Multiple locations (Fontan, Baffle and } \\
\text { fenestration, DVT in leg, iliac, femoral } \\
\text { veins, and so forth) }\end{array}$ & 2 \\
\hline Left ventricle & 1 \\
\hline Right atrium & 1 \\
\hline Shunt thrombosis & 1 \\
\hline Stroke & 1 \\
\hline Sagittal sinus thrombosis & 1 \\
\hline
\end{tabular}

Continuous variables are presented as the median with interquartile range and categorical variables are expressed as a percentage. TAPVR, Total anomalous pulmonary venous return; $P A$, pulmonary artery; $L V O T$, left ventricular outflow tract; $A L C A P A$, anomalous origin of the left coronary artery from the pulmonary artery; $D V T$, deep vein thrombosis.

and thrombosis $(P<.05)$. A change in aspirin dose after aspirin testing occurred in 4 patients. Repeat testing was performed in only 2 of these patients, demonstrating conversion from unresponsiveness to adequate response. In the other 2 patients who did not undergo repeat testing, the aspirin dose was altered after a thrombosis event. Among 28 patients receiving heparin at the time of testing, $3(10.7 \%)$ developed thrombosis, and the risk of thrombosis was not significantly different compared with the entire cohort.

\section{DISCUSSION}

This study demonstrates significant variability in platelet inhibition after administration of aspirin according to current practice patterns at a single institution. Our study suggests approximately $10 \%$ aspirin unresponsiveness in the entire cohort, and more than $50 \%$ of neonates undergoing complex cardiac surgical procedures are unresponsive to an aspirin dosage of $20.25 \mathrm{mg} / \mathrm{d}$. In pediatric patients undergoing high-risk congenital cardiac surgical procedures involving insertion of prosthetic material into the circulation or coronary artery reconstruction, the thrombosis rate was $7.4 \%$ within 30 days of surgery, and aspirin unresponsiveness was associated with a higher risk of thrombosis.

This study demonstrates significant variability in response to a standard dose of aspirin in infants $(3-5 \mathrm{mg} / \mathrm{kg} / \mathrm{d}){ }^{8}$ Variability in response to aspirin, particularly in neonates and infants after cardiac surgery, has been demonstrated previously. ${ }^{4}$ These data highlight the importance of measuring platelet response if therapeutic efficacy is desired. Unresponsiveness was particularly high $(57 \%)$ in neonates who were given $20.25 \mathrm{mg} / \mathrm{d}(5-10 \mathrm{mg} / \mathrm{kg} / \mathrm{d})$. Similarly, patients greater than 17 years of age and $80 \mathrm{~kg}$ body weight who were given $81 \mathrm{mg} / \mathrm{d}$ of aspirin had a relatively high rate of unresponsiveness ( 1 in 3 ). Current practice in our institution is to administer $81 \mathrm{mg}$ to adolescents, and unresponsiveness in this group may be related to an aspirin dose of less than $1 \mathrm{mg} / \mathrm{kg} / \mathrm{d}$.

This study was unable to determine whether unresponsiveness was related to inadequate dosing or true aspirin resistance, which implies failure to inhibit platelet function even at maximal dose. Two infants who were unresponsive at 20.25 and $40.5 \mathrm{mg} / \mathrm{d}$ became responsive after the aspirin dose was increased, suggesting inadequate dosing as the mechanism of unresponsiveness in a subset of patients. Previous studies have suggested that the incidence of true aspirin resistance in children is approximately $2 \%$ and that many patients respond to an appropriate increase in aspirin dosing. ${ }^{4,9}$ Future studies should determine if dose adjustment, particularly in neonates and pediatric patients greater than $80 \mathrm{~kg}$, improves responsiveness.

The ability of platelet response to aspirin therapy (as a single agent) to predict thrombotic events has not been consistently demonstrated in adults, whereas response to aspirin plus clopidogrel has been shown to predict ischemic events. ${ }^{4,10-14}$ There are few reports of data correlating aspirin responsiveness with thrombosis events in children. One study on 50 children after cardiac surgery concluded a lack of correlation. ${ }^{15}$ One possible reason for the disparate outcomes compared with our study may be related to patient selection; our cohort included patients who were considered to be at the highest risk for thrombosis and excluded lowrisk patients from the analysis, whereas the previous study included even low-risk patients. Another difference may be the methodology used for detection of thrombosis; in our study, all patients had echocardiography within 2 weeks of surgery in addition to clinical assessment, whereas the 
TABLE 2. Relationship between aspirin dose and unresponsiveness or thrombosis

\begin{tabular}{|c|c|c|c|}
\hline Variable & $20.25 \mathrm{mg} / \mathrm{d}$ & $40.5 \mathrm{mg} / \mathrm{d}$ & $81 \mathrm{mg} / \mathrm{d}$ \\
\hline Number of patients & 7 & 50 & 38 \\
\hline Age & 3 wk (2-4 wk) & $5.3 \mathrm{mo}(1.7 \mathrm{mo}$ to $1.1 \mathrm{y})$ & 3.25 y $(2.4-10.8 \mathrm{y})$ \\
\hline Weight $(\mathrm{kg})$ & $3(2.6-3.5)$ & $5.1(4-7.3)$ & $15.7(12.3-28.9)$ \\
\hline Aspirin unresponsiveness, $\mathrm{n}(\%)$ & $4(57.1)^{*}$ & $4(8)$ & $2(5.3)$ \\
\hline Thrombosis, n (\%) & $2(28.6) \dagger$ & $3(6)$ & $2(5.3)$ \\
\hline
\end{tabular}

*Significantly different unresponsiveness to aspirin dose of $20.25 \mathrm{mg} / \mathrm{d}$ versus $40.5 \mathrm{mg} / \mathrm{d}(P=.005)$ and $20.25 \mathrm{mg} / \mathrm{d}$ versus $81 \mathrm{mg} / \mathrm{d}(P=.003)$. †Significantly different rate of thrombosis to aspirin dose of $20.25 \mathrm{mg} / \mathrm{d}$ versus $81 \mathrm{mg} / \mathrm{d}(P=.025)$ but not $20.25 \mathrm{mg} / \mathrm{d}$ versus $40.5 \mathrm{mg} / \mathrm{d}(P=.06)$. Continuous variables are presented as the median with interquartile range and comparisons were made using the independent Student $t$ test. Categorical variables are expressed as a percentage and compared using the Fisher exact test.

previous study relied primarily on clinical presentation for assessment of thrombosis events. Given the conflicting data regarding the relationship between responsiveness and thrombosis, a larger study will be necessary to confirm the results of this study.

An association between aspirin unresponsiveness and thrombosis has several potential clinical implications. The most important implication is that adjustment of aspirin dose or the addition of an alternative antiplatelet or anticoagulant agent may prevent thrombosis in unresponsive patients. However, this hypothesis was not tested in our study and warrants assessment in a prospective clinical trial. Although escalation of therapy in unresponsive patients may reduce the overall rate of thrombosis, it may also increase the rate of other adverse events, such as bleeding, Reyes syndrome, gastrointestinal complications, further emphasizing the importance of a trial to systematically assess therapeutic impact. The implication that platelet reactivity testing should be performed routinely after high-risk cardiac surgery in pediatric populations is based on demonstration of clinical benefit of testing-based therapeutic adjustment. Although the definition of unresponsiveness ( $\geq 550$ ARU) was extrapolated from adult

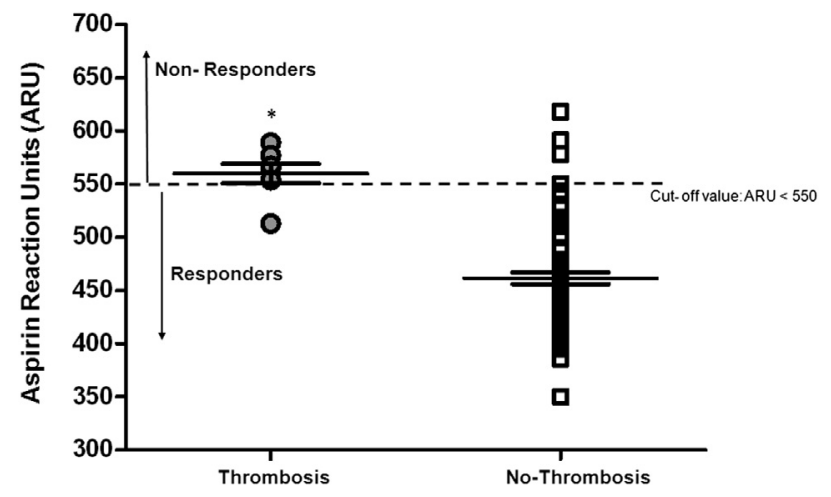

FIGURE 1. Association between aspirin unresponsiveness and thrombosis: Circles represent patients with thrombosis and squares represent patients without thrombosis. Dashed line indicates the cut-off value for aspirin unresponsiveness ( $\geq 550$ aspirin reaction units $[A R U])$. *Significant difference in aspirin unresponsiveness between patients with thrombosis compared with those without thrombosis. practice, this value provided a significant discrimination in clinical behavior in this cohort. Further validation in a larger trial is necessary to determine the most appropriate inflection point for pediatric populations. Adjustment of the threshold value based on clinical data may allow optimization of the sensitivity and specificity of the test to predict future thrombosis events.

Our choice of platform for platelet reactivity testing was based on previous experience and the existing literature. In recent years, several point-of-care platelet function testing analyzers have emerged. Currently available systems include VerifyNow, TEG-5000 Thrombelastograph (Haemoscope Corporation, Niles, Ill), Multiplate analyzer (Roche Diagnostics, Indianapolis, Ind), PFA-100 platelet function assay (Dade Behring, Newark, Del) and Plateletworks (Helena Laboratories, Beaumont, Tex) ${ }^{16-20}$ Because of significant variability in the results obtained from different analyzers, the prevalence of aspirin unresponsiveness varies widely $(5 \%-51 \%)$ between studies. ${ }^{20}$ Among these, the VerifyNow analyzer seems to be the preferred system because it uses arachidonic acid (a direct activator of cyclooxygenase-1) as the agonist, whereas others use adenosine diphosphate to assess aspirin responsiveness. ${ }^{17,19,21}$

The rate of thrombosis of $7.4 \%$ documented in this study is lower than previously reported. ${ }^{3-5}$ The lower rate detected may be attributed to exclusion of patients presenting with thrombosis before initiation of therapy. Similarly, although $60 \%$ of patients who were unresponsive to aspirin therapy had a thrombosis event, this number may not reflect the true incidence of thrombosis in unresponsive patients. In 2 patients who were found to be unresponsive on initial testing, the aspirin dose was increased by the clinical team (who were blinded to the platelet function results), but repeat testing was not performed. If these patients who did not have thrombosis responded to the increased dose of aspirin, then the result would further bolster the findings of this study.

There are several limitations to this single-center pilot study. First, the number of patients and thrombosis events studied is small, limiting its power. Verification in a larger cohort is necessary to confirm the results. Second, this study 
terminated follow-up at 30 days after surgery, but longerterm follow-up is more relevant, particularly because a significant proportion of thrombosis-related complications occur beyond the 30-day time frame. Third, the incidence of thrombosis may be underestimated by this study because echocardiography does not detect peripheral vessel thrombosis.

In conclusion, this study demonstrates that aspirin unresponsiveness occurs in approximately $10.4 \%$ of patients undergoing specific high-risk congenital cardiac procedures using the dosing practice of a single institution. Aspirin unresponsiveness as measured by the VerifyNow system predicts the 30-day risk of thrombosis in patients after specific complex cardiac surgical procedures. Future studies are necessary to demonstrate if monitoring of aspirin therapy with consequent dose adjustment or addition of alternative agents for unresponsive patients minimizes the risk of thrombosis in this cohort.

\section{References}

1. Andrew ME, Monagle P, deVeber G, Chan AK. Thromboembolic disease and antithrombotic therapy in newborns. Hematology Am Soc Hematol Educ Program. 2001;358-74.

2. Gruenwald CE, Manlhiot C, Crawford-Lean L, Foreman C, Brandao LR, McCrindle BW, et al. Management and monitoring of anticoagulation for children undergoing cardiopulmonary bypass in cardiac surgery. J Extra Corpor Technol. 2010:42:9-19.

3. Emani S, Zurakowski D, Baird CW, Pigula FA, Trenor C III, Emani SM. Hypercoagulability markers predict thrombosis in single ventricle neonates undergoing cardiac surgery. Ann Thorac Surg. 2013;96:651-6.

4. Romlin BS, Wahlander H, Stromvall-Larsson E, Synnergren M, Baghaei F, Jeppsson A. Monitoring of acetyl salicylic acid-induced platelet inhibition with impedance aggregometry in children with systemic-to-pulmonary shunts. Cardiol Young. 2013;23:225-32.

5. Manlhiot C, Menjak IB, Brandao LR, Gruenwald CE, Schwartz SM, Sivarajan VB, et al. Risk, clinical features, and outcomes of thrombosis associated with pediatric cardiac surgery. Circulation. 2011;124:1511-9.

6. Monagle P, Cochrane A, Roberts R, Manlhiot C, Weintraub R, Szechtman B, et al. A multicenter, randomized trial comparing heparin/warfarin and acetylsalicylic acid as primary thromboprophylaxis for 2 years after the Fontan procedure in children. J Am Coll Cardiol. 2011;58:645-51.

7. Heistein LC, Scott WA, Zellers TM, Fixler DE, Ramaciotti C, Journeycake JM. Aspirin resistance in children with heart disease at risk for thromboembolism: prevalence and possible mechanisms. Pediatr Cardiol. 2008;29:285-91.

8. Israels SJ, Michelson AD. Antiplatelet therapy in children. Thromb Res. 2006; 118:75-83.

9. Yee DL, Dinu BR, Sun CW, Edwards RM, Justino H, Teruya J, et al. Low prevalence and assay discordance of "aspirin resistance" in children. Pediatr Blood Cancer. 2008;51:86-92.

10. Stone GW, Witzenbichler B, Weisz G, Rinaldi MJ, Neumann FJ, Metzger DC, et al. Platelet reactivity and clinical outcomes after coronary artery implantation of drug-eluting stents (ADAPT-DES): a prospective multicentre registry study. Lancet. 2013;382:614-23.

11. Lee SP, Park KW, Shin DH, Lee HY, Kang HJ, Koo BK, et al. Efficacy of predicting thrombotic events with combination of dual point-of-care testing (POCT) after drug-eluting stent implantation for coronary heart disease: results from the CILON-T randomized trial POCT substudy. J Atheroscler Thromb. 2011;18:914-23.

12. Pinto Slottow TL, Bonello L, Gavini R, Beauzile P, Sushinsky SJ, Scheinowitz M, et al. Prevalence of aspirin and clopidogrel resistance among patients with and without drug-eluting stent thrombosis. Am J Cardiol. 2009; 104:525-30.

13. Reny JL, De Moerloose P, Dauzat M, Fontana P. Use of the PFA-100 closure time to predict cardiovascular events in aspirin-treated cardiovascular patients: a systematic review and meta-analysis. J Thromb Haemost. 2008;6:444-50.
14. Collet JP, Cuisset T, Range G, Cayla G, Elhadad S, Pouillot C, et al. Bedside monitoring to adjust antiplatelet therapy for coronary stenting. $N$ Engl J Med. 2012;367:2100-9.

15. Cholette JM, Mamikonian L, Alfieris GM, Blumberg N, Lerner NB. Aspirin resistance following pediatric cardiac surgery. Thromb Res. 2010;126: 200-6.

16. Breet NJ, van Werkum JW, Bouman HJ, Kelder JC, Ruven HJ, Bal ET, et al. Comparison of platelet function tests in predicting clinical outcome in patients undergoing coronary stent implantation. JAMA. 2010;303:754-62.

17. Kovács EG, Katona É, Bereczky Z, Homoródi N, Balogh L, Tóth E, et al. Evaluation of laboratory methods routinely used to detect the effect of aspirin against new reference methods. Thromb Res. 2014;133:811-6.

18. Lordkipanidze M, Pharand C, Schampaert E, Turgeon J, Palisaitis DA, Diodati JG. A comparison of six major platelet function tests to determine the prevalence of aspirin resistance in patients with stable coronary artery disease. Eur Heart J. 2007;28:1702-8.

19. Michelson AD. Methods for the measurement of platelet function. Am J Cardiol. 2009; 103(3 Suppl):20A-6A.

20. Sambu N, Curzen N. Monitoring the effectiveness of antiplatelet therapy: opportunities and limitations. Br J Clin Pharmacol. 2011;72:683-96.

21. Cheung EW, Ho SA, Tang KK, Chau AK, Chiu CS, Cheung YF Pericardial effusion after open heart surgery for congenital heart disease. Heart. $2003 ; 89: 780-3$

\section{Discussion}

Dr Jennifer Hirsch-Romano (Ann Arbor, Mich). Thank you, Ram, for an excellent presentation. You really highlight a significant problem in the congenital heart disease population. Thromboses in these patients can span from being just challenging peripheral venous thromboses that make central access incredibly difficult as these patients age to fatal shunt thrombosis. I think the understanding of aspirin resistance is really new to our field and we are just getting new information with studies such as yours. I am hoping that with a better understanding of aspirin resistance as well as various antithrombotic agents, we will get a better understanding of how to manage our patients.

My first question to you is: in all of our neonates, we routinely use a 40.5-mg dose. I am interested to know whether the high rate of aspirin unresponsiveness as well as the high thrombosis rate in your neonates have led to changes in any of the practice management in your own institution in terms of that dosing.

Dr Emani. A proposed algorithm reflecting the findings of our study includes the use of a higher dose of $40.5 \mathrm{mg}$ aspirin in neonates greater than $3 \mathrm{~kg}$. We are hesitant to use $40.5 \mathrm{mg}$ in patients less than $2.5 \mathrm{~kg}$. But we have started using $40 \mathrm{mg}$ more commonly in the neonates. The data would support being more aggressive about testing neonates and then considering a dose adjustment if unresponsive.

Dr Hirsch-Romano. My next question has to do with the screening you did within your study. I know you specifically did echo monitoring for intracardiac thromboses and otherwise followed for clinical events. Have you considered doing any routine screening for peripheral thromboses? Quite often the loss of venous access in these children is silent without obvious signs of limb ischemia.

Dr Emani. I think we need to be more thorough in our assessment of peripheral vascular thrombosis, which is under-reported. In this study, only clinically significant events such as limb ischemia were used to define peripheral thrombosis. Imaging studies are suboptimal at detecting asymptomatic events. I think 
moving forward, we are going to have to be much more systematic about imaging and detecting these types of events. Fortunately, the methodology was consistent among all patients, so although we may be underestimating the incidence of thrombosis, the relationship of thrombosis to aspirin unresponsiveness still holds true.

Dr Hirsch-Romano. Then my final question has to do with the use of Plavix. I think the use of Plavix in the pediatric population is still a bit controversial, but it is certainly increasing at many congenital heart centers. Are you using Plavix in your pediatric population; and if so, for what indications?

Dr Emani. Plavix, as you know, also has its downsides. About $20 \%$ to $25 \%$ of patients are Plavix unresponsive, and the bleeding risk is higher. But I would certainly use Plavix for a patient who is at high risk for thrombosis and is resistant to aspirin.

According to our proposed algorithm, in a patient who is unresponsive, if you really want to understand if is this an absorption issue versus inadequate dosing, you can actually spike the blood with a maximum dose of aspirin to determine true resistance. I think in a truly resistant patient I would use Plavix, but would test for the effect of Plavix as well.

Dr Hirsch-Romano. Can you tell me about your VerifyNow testing? I know historically when we had a look at various testing options, quite often they require a fair blood volume, which is challenging in a neonatal population, or they require a nontourniquet blood draw, which also can be challenging.

Dr Emani. It requires $2 \mathrm{~mL}$ of blood and that is a major issue. We are working with a couple of different systems, microfluidic systems, to try to reduce that volume.

A major problem with platelet testing, particularly if you use small-volume draws, is the hypersensitive nature of the clotting system after introducing any foreign object into the blood stream. So a finger stick or a small-volume blood draw is likely to be contaminated by the contact activation. So novel methods of in vivo platelet reactivity testing need to be developed to overcome this limitation.

Dr Henry Walters (Detroit, Mich). This was an excellent presentation. Jenna, you had mentioned the catastrophic situation in which there is thrombosis of a systemic-to-pulmonary artery shunt. If we are still in the mood for surveys, I was wondering if we could conduct another one.

Dr Carl L. Backer (Chicago, Ill). We are in the mood. Go ahead.

Dr Walters. Great. Well, I am going to leave the survey questions up to you, but, for example, after constructing a systemic-to-pulmonary artery shunt, 1 program might start with an intravenous heparin infusion and transition to some kind of antiplatelet agent. Others might use aspirin only, Plavix only, or a combination. I would be interested in how the members of this audience approach this situation.

Dr Backer. Dr Hirsch-Romano has already read your mind and I have got a list here.

For a neonate having a modified Blalock-Taussig shunt (our biggest fear I think for thrombosis), how many surgeons would use aspirin only?

(Show of hands.)

It looks like about a third of the hands.

And how many would use Plavix only? Nobody is using Plavix only.
How about aspirin plus Plavix?

Nobody. There is no Plavix.

What about heparin, intravenous heparin therapy?

Dr Hirsch-Romano. Heparin bridge to aspirin.

Dr Backer. Heparin bridge to aspirin.

(Show of hands.)

Oh, boy, now everybody's hands are going up. We have got about $80 \%$ of the audience.

How about Lovenox, injections of Lovenox; who uses Lovenox?

(Show of hands.)

Only 3 hands for Lovenox.

It seems that most surgeons when they are really concerned about thrombosis are using heparin followed by aspirin. What are your thoughts about that Ram?

Dr Emani. I use heparin followed by aspirin, partly because I do not think there is a great antiplatelet agent for the immediate postoperative period. When we looked at shunt thrombosis in particular, it is actually white thrombus suggesting platelet aggregation rather than thrombosis mediated by the coagulation system, so an antiplatelet agent is actually what you need. The question is whether you can do that safely and effectively with current therapeutic options. But I still use heparin and try to get it therapeutic before initiation of aspirin.

Dr Sabine Daebritz (Duisburg, Germany). It was an interesting talk. Did you screen your patients for any other reasons for thrombosis like factor V Leiden or heparin-induced thrombocytopenia? This is important because some of those thromboses, like sagittal sinus thrombosis, are not considered to be influenced much by aspirin so they might be caused by something else.

Dr Emani. Yes, many of the patients with thrombosis are screened for hypercoagulability disorders as well. Some of the neonates and single-ventricle patients who do not have thrombosis were screened with a thrombophilia panel as a part of a separate study. We have found that the presence of a hypercoagulability disorder increases the risk of a thrombosis event. The patient who had sagittal sinus thrombosis particularly did not have a coagulation abnormality, nor did any of the other patients who had thrombosis. We did not systematically screen all patients without thrombosis.

But I would add that even if thrombophilia panels come back negative, it does not mean that a child does not have some form of hypercoagulability disorder or platelet hyperreactivity. I would argue that all high-risk patients should therefore be aggressively managed with anticoagulation or antiplatelet therapy?

Dr Joseph J. Amato (Chicago, Ill). I was just curious, when you were doing the diagnosis of atrial septal defect, you mentioned that a certain percentage, a small percentage, were looked at with catheterization. Is that a necessity or it just so happened?

Dr Emani. The patients who underwent catheterization for hemodynamic reasons would have been followed for a thrombosis event. So we have looked at those images to see if thrombosis was present as an incidental finding. None of the patients had thrombosis detected by catheterization alone.

Dr Amato. You could have defined them by just echo alone?

Dr Emani. Right. Most of the catheterizations that occurred were performed for hemodynamic reasons rather than confirmation of echo. 
Dr J. William Gaynor (Philadelphia, Pa). Ram, I enjoyed that. One quick question. There are obviously multiple factors that can play into the risk of thrombosis, and 1 of those is the shunt itself. This is completely anecdotally, but a few years ago we switched to the heparin-bonded shunts and it seems like our rate of thrombosis (we still use heparin as a bridge to aspirin), but it seems like our rate of early shunt thrombosis has decreased with that. We have not looked at it systemically. It is anecdotal. I was wondering what shunt material you used?

Dr Emani. Well, I was excited when the heparin-bonded shunt came out and I use that on all of my patients and I have not found a difference.

The patients who develop thrombosis of polytetrafluoroethylene material demonstrate platelet-mediated occlusion rather than coagulation-mediated. So I am not sure if the heparin helps much. As we start to understand the clotting system in general, I think we can start to understand that there are actually therapies in each of the systems; the coagulation platelet and fibrinolytic systems. Hopefully as we collect more data, we can tailor our approach based on what we think the predominant deficits are. We need better monitoring systems and better therapeutic options.

Dr Backer. Bill, you bring up a good point. Let me survey the audience. How many surgeons have switched to using the heparinbonded shunts?

(Show of hands.)

And how many are still using the non-heparin-bonded shunts? (Show of hands.)

Looks like about 50/50 or maybe 60/40 for the heparin-bonded shunts. Our group has certainly switched to them, and at least anecdotally I have found that we have had less shunt thrombosis. 